PROCEEDINGS OF THE

AMERICAN MATHEMATICAL SOCIETY

Volume 126, Number 4, April 1998, Pages 1211-1218

S 0002-9939(98)04163-X

\title{
ON STONE'S THEOREM AND THE AXIOM OF CHOICE
}

\author{
C. GOOD, I. J. TREE, AND W. S. WATSON \\ (Communicated by Andreas R. Blass)
}

\begin{abstract}
It is a well established fact that in Zermelo-Fraenkel set theory, Tychonoff's Theorem, the statement that the product of compact topological spaces is compact, is equivalent to the Axiom of Choice. On the other hand, Urysohn's Metrization Theorem, that every regular second countable space is metrizable, is provable from just the ZF axioms alone. A. H. Stone's Theorem, that every metric space is paracompact, is considered here from this perspective. Stone's Theorem is shown not to be a theorem in ZF by a forcing argument. The construction also shows that Stone's Theorem cannot be proved by additionally assuming the Principle of Dependent Choice.
\end{abstract}

\section{INTRODUCTION}

Given an infinite set $X$, is it possible to define a Hausdorff topology on $X$ such that $X$ has at least two non-isolated points? In ZFC, the answer is easily shown to be yes. However, models of $\mathrm{ZF}$ exist that contain infinite sets that cannot be expressed as the union of two disjoint infinite sets (amorphous sets - see [6]). For any model of ZF containing an amorphous set, the answer is no. So, as we see, even the most innocent of topological questions may be undecidable from the ZermeloFraenkel axioms alone. Further examples of the counter-intuitive behaviour of 'choiceless topology' can be found in [3] and [4].

The concept of paracompactness in a topological space was first defined by Dieudonné in [2], in which he proved that every metrizable space that is second countable or locally compact is paracompact. The importance of paracompactness in general topology was raised when A. H. Stone proved the theorem of the title, namely that every metric space is paracompact [11]. Mary Ellen Rudin later improved the proof in [10]. One notable point about Rudin's proof is the very first line: 'Let $\mathcal{U}=\left\{U_{\alpha}\right\}$ be an open cover indexed by ordinals', an immediate use of the Axiom of Choice. Stone's original proof also uses Choice, but in a less obvious manner. We are therefore prompted to ask whether this is an essential part of Stone's Theorem: just how heavily does Stone's Theorem depend on the Axiom of Choice? We address this question here.

The fact that every separable metrizable space is paracompact can be proved from ZF [4]. That every second countable metric space is paracompact, Dieudonné's original result, can also be so proved (but recall from [4] that there are models of ZF containing second countable metric spaces that are not separable). However, we show that this is not true of the general theorem: we construct symmetric models of ZF in which there are metric spaces that are not paracompact.

Received by the editors March 27, 1996 and, in revised form, September 17, 1996.

1991 Mathematics Subject Classification. Primary 54D20; Secondary 03E25. 


\section{SYMMETRIC MODELS OF ZF}

We review some facts about symmetric models, referring the reader to [6] for further details. Our notation follows that of [6] and [7].

Let $\mathcal{M}$ be a transitive model of ZFC and $B$ a complete Boolean algebra in $\mathcal{M}$. For an automorphism $\pi$ of $B$, we extend $\pi$ to $\mathcal{M}^{B}$ by induction on the rank of $x \in \mathcal{M}^{B}$ :

(1) $\pi(0)=0$

(2) $\operatorname{dom}(\pi x)=\{\pi y: y \in \operatorname{dom}(x)\}$ and $(\pi x)(\pi y)=\pi(x(y))$.

It follows that $\pi$ is a one-to-one function from $\mathcal{M}^{B}$ onto itself, and $\pi \check{x}=\check{x}$ for every $x \in \mathcal{M}$. Let $\mathcal{G}$ be a group of automorphisms of $B$. A non-empty set $\mathcal{F}$ of subgroups of $\mathcal{G}$ is called a normal filter on $\mathcal{G}$ if and only if for all subgroups $H, K$ of $\mathcal{G}$,

(i) if $K \in \mathcal{F}$ and $K \subseteq H$ then $H \in \mathcal{F}$,

(ii) if $H \in \mathcal{F}$ and $K \in \mathcal{F}$ then $H \cap K \in \mathcal{F}$,

(iii) if $\pi \in \mathcal{G}$ and $H \in \mathcal{F}$ then $\pi H \pi^{-1} \in \mathcal{F}$.

Let $\mathcal{F}$ be a fixed normal filter. For each $x \in \mathcal{M}^{B}$, define $\operatorname{sym}(x)=\{\pi \in \mathcal{F}$ : $\pi x=x\}$. We say that $x \in \mathcal{M}^{B}$ is symmetric if $\operatorname{sym}(x) \in \mathcal{F}$. The class $H S \subseteq \mathcal{M}^{B}$ of all hereditarily symmetric names is defined by recursion:

(a) $0 \in H S$;

(b) if $\operatorname{dom}(x) \subseteq H S$ and $x$ is symmetric, then $x \in H S$.

Now let $G$ be an $\mathcal{M}$-generic ultrafilter on $B$ and $i_{G}$ be the interpretation of $\mathcal{M}^{B}$ by $G$. Define $\mathcal{N}=\left\{i_{G}(x): x \in H S\right\}$. Then $\mathcal{N}$ is a symmetric extension of $\mathcal{M}$ and $\mathcal{M} \subseteq \mathcal{N} \subseteq \mathcal{M}[G]$. More importantly, $\mathcal{N}$ is a model of ZF.

\section{THE PARTIAL ORDER}

We use one partial order as the basis for our symmetric model constructions, namely $\mathbf{P}=F n\left(\omega \times \mathbf{R} \times \omega_{1} \times \omega_{1}, 2, \omega_{1}\right)$, the set of partial functions $p$ with $|\operatorname{dom}(p)|<$ $\omega_{1}, \operatorname{dom}(p) \subseteq \omega \times \mathbf{R} \times \omega_{1} \times \omega_{1}$ and $\operatorname{ran}(p)=\{0,1\}$. We define the ordering on $\mathbf{P}$ by $p \leqslant q$ if and only if $q \subseteq p$. Let $B=R O(P)$ in $\mathcal{M}$, the complete Boolean algebra of regular open sets of $\mathbf{P}$ in $\mathcal{M}$.

Define the following elements of $\mathcal{M}[G]$ together with their canonical names:

$$
\begin{aligned}
& x_{n r \alpha}=\left\{\delta \in \omega_{1}: \exists p \in G p(n, r, \alpha, \delta)=1\right\}, \\
& \operatorname{dom}\left(\underline{x}_{n r \alpha}\right)=\left\{\check{\delta}: \delta \in x_{n r \alpha}\right\}, \\
& \underline{x}_{n r \alpha}(\check{\delta})=\sup \{p \in \mathbf{P}: p(n, r, \alpha, \delta)=1\}=u_{n r \alpha \delta}, \\
& X_{n r}=\left\{x_{n r \alpha}: \alpha \in \omega_{1}\right\}, \\
& \operatorname{dom}\left(\underline{X}_{n r}\right)=\left\{\underline{x}_{n r \alpha}: \alpha \in \omega_{1}\right\}, \\
& \underline{X}_{n r}\left(\underline{x}_{n r \alpha}\right)=1, \\
& R_{n}=\left\{X_{n r}: r \in \mathbf{R}\right\}, \\
& \operatorname{dom}\left(\underline{R}_{n}\right)=\left\{\underline{X}_{n r}: r \in \mathbf{R}\right\}, \\
& \underline{R}_{n}\left(\underline{X}_{n r}\right)=1, \\
& M=\left\{R_{n}: n \in \omega\right\}, \\
& \operatorname{dom}(\underline{M})=\left\{\underline{R}_{n}: n \in \omega\right\}, \\
& \underline{M}\left(\underline{R}_{n}\right)=1 .
\end{aligned}
$$


Let $d(x, y)=|x-y|$ be the usual distance function on $\mathbf{R}$ that generates the Euclidean topology (in $\mathcal{M}$ ). Recall that if $G$ is $\mathbf{P}$-generic over $\mathcal{M}$ then $(\mathbf{R})^{\mathcal{M}}=$ $(\mathbf{R})^{\mathcal{N}}=(\mathbf{R})^{\mathcal{M}[G]}$, where $\mathcal{M} \subseteq \mathcal{N} \subseteq \mathcal{M}[G]$ - see [7, 7.6.14].

Theorem 1. Let $\mathcal{M}$ be a transitive model of ZFC. There is a symmetric extension $\mathcal{N}$ of $\mathcal{M}$ which contains a collection of sets $R_{n}$ for which there is no set $S \in \mathcal{N}$ with $\varnothing \neq S \cap R_{n} \subsetneq R_{n}$ for all $n$ and where $\bigcup R_{n}$ can be topologized as a metric space with each $R_{n}$ a connected subspace.

Proof. We define a group $\mathcal{G}$ and a filter $\mathcal{F}$ such that the names $\underline{x}_{n r \alpha}, \underline{X}_{n r}, \underline{R}_{n}$ and $\underline{M}$ are all symmetric. Observe that every permutation $\pi$ on $\omega \times \mathbf{R} \times \omega_{1}$ induces an order-preserving 1-1 mapping on $\mathbf{P}$, by $(\pi p)(\pi(n, r, \alpha), \delta)=p(n, r, \alpha, \delta)$, and an automorphism of $B$, by $\pi u=\sup \{\pi p: p \leqslant u\}$. One can check that

$$
\pi\left(u_{n r \alpha \delta}\right)=u_{\pi(n, r, \alpha) \delta} \text { and } \pi\left(\underline{x}_{n r \alpha}\right)=\underline{x}_{\pi(n, r, \alpha)} \cdots
$$

Let $\mathcal{G}$ be the group of all automorphisms of $B$ induced by permutations of $\omega \times \mathbf{R} \times \omega_{1}$ satisfying $\pi(n, r, \alpha)=\left(n, \rho(r), \alpha^{\prime}\right)$, where $\pi(n, r,$.$) is a permutation on \omega_{1}$ for fixed $n, r$ and $\rho=\rho^{\pi}: \mathbf{R} \rightarrow \mathbf{R}$ is either a reflection about some point $x_{\pi} \in \mathbf{R}$, or the identity map.

By $(\dagger), \operatorname{dom}\left(\pi \underline{X}_{n t}\right)=\left\{\underline{x}_{\pi(n, t, \alpha)}: \alpha \in \omega_{1}\right\}=\operatorname{dom}\left(\underline{X}_{n \rho(t)}\right)$. It follows that, as $\pi \in \mathcal{G}$ is an automorphism of $B, \pi\left(\underline{R}_{n}\right)=\underline{R}_{n}$ and $\pi(\underline{M})=\underline{M}$.

For each finite subset $e \subseteq \omega \times \mathbf{R} \times \omega_{1}$, let $f i x(e)=\{\pi \in \mathcal{G}: \forall s \in e \pi(s)=s\}$. Let $\mathcal{F}$ be the filter on $\mathcal{G}$ generated by $\left\{\right.$ fix $\left.(e): e \in\left[\omega \times \mathbf{R} \times \omega_{1}\right]^{<\omega}\right\}$.

Claim 1.1. $\mathcal{F}$ is a normal filter on $\mathcal{G}$.

Proof. Omitted.

Let $H S$ be the set of all hereditarily symmetric names in $\mathcal{M}^{B}$. Let $\mathcal{N}$ be the symmetric extension of $\mathcal{M}$ given by the interpretation of $H S$ by $G$.

Claim 1.2. For all $n, r$ and $\alpha$, the sets $x_{n r \alpha}, X_{n r}, R_{n}, M$ are in the model $\mathcal{N}$.

Proof. By $(\dagger), \operatorname{sym}\left(\underline{x}_{n r \alpha}\right)=f i x((n, r, \alpha)) \in \mathcal{F}$. Inductively, $\check{\delta} \in H S$ for all $\delta \in \omega_{1}$, so $\operatorname{dom}\left(\underline{x}_{n r \alpha}\right) \subseteq H S$. Hence $\underline{x}_{n r \alpha} \in H S$. Fix $\alpha_{0} \in \omega_{1}$. Then

$$
\begin{aligned}
\operatorname{sym}\left(\underline{X}_{n r}\right)= & \left\{\pi \in \mathcal{G}: \pi \underline{X}_{n r}=\underline{X}_{n r}\right\} \\
= & \left\{\pi: \operatorname{dom}\left(\pi \underline{X}_{n r}\right)=\operatorname{dom}\left(\underline{X}_{n r}\right)\right. \\
& \left.\quad \text { and } \pi \underline{X}_{n r}\left(\pi \underline{x}_{n r \alpha}\right)=\pi\left(\underline{X}_{n r}\left(\underline{x}_{n r \alpha}\right)\right)=\pi(1)=1\right\} \\
& \supseteq f i x\left(n, r, \alpha_{0}\right) .
\end{aligned}
$$

So $\operatorname{sym}\left(\underline{X}_{n r}\right) \in \mathcal{F}$ and $\operatorname{dom}\left(\underline{X}_{n r}\right) \subseteq H S$. Thus each $\underline{X}_{n r}$ is in $H S$. It follows that $\operatorname{dom}\left(\underline{R}_{n}\right) \subseteq H S$ and $\operatorname{sym}\left(\underline{R}_{n}\right)=\mathcal{G}$, so $\operatorname{dom}(\underline{M}) \subseteq H S$ and $\operatorname{sym}(\underline{M})=\mathcal{G}$. Therefore each $\underline{R}_{n}$ and $\underline{M}$ is in $H S$. By the definition of the interpretation of $\mathcal{M}^{B}$ by $G$, we have $x_{n r \alpha}, X_{n r}, R_{n}, M \in \mathcal{N}$.

Claim 1.3. There is no function $f \in \mathcal{N}$ such that $\operatorname{dom}(f)=M$ and $f\left(R_{n}\right)$ is a proper non-empty subset of $R_{n}$ for each $n$.

Proof. Assume there is such an $f \in \mathcal{N}$. Let $\underline{f}$ be a symmetric name for $f$ and let $p_{0} \in G$ be such that

$$
p_{0} \Vdash(\underline{f} \text { is a function }) \text { and }\left(\forall \check{n} \varnothing \neq \underline{f}\left(\underline{R}_{n}\right) \subsetneq \underline{R}_{n}\right) .
$$


Let $e$ be a finite subset of $\omega \times \mathbf{R} \times \omega_{1}$ such that $f i x(e) \subseteq \operatorname{sym}(f)$. Pick $n \in \omega$ such that $e \cap\left(\{n\} \times \mathbf{R} \times \omega_{1}\right)=\varnothing$. Then there are $r, s \in \mathbf{R}$ and $p \leqslant p_{0}$ such that

$$
p \Vdash \underline{X}_{n r} \in \underline{f}\left(\underline{R}_{n}\right) \text { and } \underline{X}_{n s} \notin \underline{f}\left(\underline{R}_{n}\right) \text {. }
$$

Fix these $n, r, s$ and $p$. Pick $\epsilon \in \omega_{1}$ such that for all $\alpha \geqslant \epsilon$ and all $t$ and $\delta$, $(n, t, \alpha, \delta) \notin \operatorname{dom}(p)$. Let $\rho$ be the reflection of $\mathbf{R}$ about the point $\frac{r+s}{2}$, so $\rho(r)=s$ and $\rho(s)=r$. The sets of ordinals $[0, \epsilon]$ and $(\epsilon, 2 \epsilon]$ are order isomorphic. Let $\phi$ be the order isomorphism: $\phi(0)=\epsilon+1, \phi(\epsilon)=2 \epsilon, \phi(\omega)=\epsilon+\omega$ and so on, and let $\pi \in \mathcal{G}$ be the permutation on $\omega \times \mathbf{R} \times \omega_{1}$ defined by

$$
\pi(m, t, \alpha)= \begin{cases}(m, t, \alpha), & m \neq n, \\ (n, \rho(t), \phi(\alpha)), & m=n, \alpha \in[0, \epsilon], \\ \left(n, \rho(t), \phi^{-1}(\alpha)\right), & m=n, \alpha \in(\epsilon, 2 \epsilon], \\ (n, \rho(t), \alpha), & m=n, \alpha>2 \epsilon .\end{cases}
$$

Then $\pi$ has the following properties:

(i) $\pi \underline{f}=\underline{f}$-after all, by the definition of $e$ and the choice of $n, \pi \in f i x(e)$.

(ii) $\pi \underline{X}_{n t}=\underline{X}_{n \rho(t)}$.

(iii) $\pi p$ and $p$ are compatible elements of $\mathbf{P}$. If $(m, t, \alpha, \delta) \in \operatorname{dom}(p) \cap \operatorname{dom}(\pi p)$ for $m \neq n$, then $(\pi p)(m, t, \alpha, \delta)=p(m, t, \alpha, \delta)$ by definition of $\pi$. If $(n, t, \alpha, \delta) \in$ $\operatorname{dom}(p) \cap \operatorname{dom}(\pi p)$, then $\left(\pi^{-1}(n, t, \alpha), \delta\right) \in \operatorname{dom}(p), \pi p(n, t, \alpha, \delta)=p\left(\pi^{-1}(n, t, \alpha), \delta\right)$ and $\alpha<\epsilon$. But $\pi^{-1}(n, t, \alpha)=\left(n, \rho(t), \phi^{-1}(\alpha)\right)$, so

$$
\pi p(n, t, \alpha, \delta)=p\left(\pi\left(n, \rho(t), \phi^{-1}(\alpha)\right), \delta\right)=p(n, t, \alpha, \delta) .
$$

To establish the Claim, notice that, by (iii), $\pi p \cup p$ is a well-defined extension of $p$. But $\pi p \Vdash \pi\left(\underline{X}_{n r}\right) \in \pi f\left(\pi\left(\underline{R}_{n}\right)\right)$ and $\pi\left(\underline{X}_{n s}\right) \notin \pi f\left(\pi\left(\underline{R}_{n}\right)\right)$. So, by (i) and (ii), $\pi p \Vdash \underline{X}_{n s} \in \underline{f}\left(\underline{R}_{n}\right)$ and $\underline{X}_{n r} \notin \underline{f}\left(\underline{R}_{n}\right)$. Therefore

$$
\pi p \cup p \Vdash\left(\underline{X}_{n s} \notin \underline{f}\left(\underline{R}_{n}\right)\right) \text { and }\left(\underline{X}_{n s} \in \underline{f}\left(\underline{R}_{n}\right)\right) .
$$

This is a contradiction.

The reader should observe from Claim 1.3 that functions such as $f\left(R_{n}\right)=\left\{X_{n 0}\right\}$, where 0 is the additive identity on $\mathbf{R}$, have no symmetric name and hence are not in $\mathcal{N}$.

Claim 1.4. $U R_{n}$ can be given a metrizable topology where each $R_{n}$ is connected.

Proof. Consider the following elements of $\mathcal{M}[G]$ :

$$
\begin{aligned}
d_{n} & =\left\{\left(X_{n r}, X_{n s}, \frac{d(r, s)}{1+d(r, s)}\right): r, s \in \mathbf{R}\right\}, \\
D & =\left\{d_{n}: n \in \omega\right\}, \\
E & =\left\{\left(X_{n r}, X_{m s}, 1\right): n \neq m \text { and } r, s \in \mathbf{R}\right\} .
\end{aligned}
$$

One can check that each of these sets has a symmetric name, and hence they are elements of $\mathcal{N}$. $E \cup \bigcup D$ defines the required metric on $\bigcup R_{n}$ in $\mathcal{N}$. This completes the proof of Theorem 1.

We now use the collection $M \in \mathcal{N}$ to construct a space contradicting Stone's Theorem.

Theorem 2. It is consistent relative to ZF that there is a (locally connected, locally compact) metric space that is not paracompact. 
Proof. Let $M=\left\{R_{n}: n \in \omega\right\}$ be the collection of sets constructed in Theorem 1. As above, $X=\bigcup M$ is a metric space (the reader may like to check that it is locally compact and to compare this with the results of [2]). We show that $X$ is not paracompact.

Define an open cover $\mathcal{U}=\left\{B_{\epsilon}(x): x \in R_{n}, \epsilon \in \mathbf{R}\right.$ and $\left.n \in \omega\right\}$. Suppose $\mathcal{U}$ had a locally finite open refinement, $\mathcal{V}$. Let $S=\{x \in X: \forall V \in \mathcal{V} x \notin \bar{V}-V\}$ and, for $n \in \omega$, let $S_{n}=S \cap R_{n}$. We claim that $S_{n}$ is a proper subset of every $R_{n}$.

Pick any $x \in R_{n}$ and some open set $W$ such that $x \in W$ and $W$ meets only finitely many elements of $\mathcal{V}$. Suppose that $W$ meets precisely the sets $V_{0}, V_{1}, \ldots, V_{k} \in \mathcal{V}$. Define a finite $F \subseteq \omega$ inductively by $i \in F$ if and only if $W \cap V_{i} \cap \bigcap\left\{V_{j}: j<i, j \in\right.$ $F\} \neq \varnothing$. Let $O=W \cap \bigcap_{i \in F} V_{i}$. Then, for $V \in \mathcal{V}, V \cap O \neq \varnothing$ if and only if $V=V_{i}$ for some $i \leqslant k$. Hence $(\bar{V}-V) \cap O=\varnothing$ for all $V \in \mathcal{V}$, i.e. $\varnothing \neq O \subseteq S_{n}$. Also, for any $V \in \mathcal{V}$ with $V \subseteq R_{n}, V$ is an open bounded subset of $R_{n}$. As $R_{n}$ is connected, there is some $z \in \bar{V}-V$, i.e. $z \notin S_{n}$.

Hence we have shown that if $\mathcal{U}$ has a locally finite open refinement, $S$ has a proper intersection with each $R_{n}$, contrary to the property of $M$.

We record here that the Principle of Dependent Choice (DC) holds in our model, demonstrating that Stone's Theorem cannot be proved from ZF + DC:

Principle of Dependent Choice. If $R$ is a relation on a set $X$ such that for all $x \in X$ there exists $y \in X$ with $x R y$, then for any $\zeta \in X$ there exists a sequence $f: \omega \rightarrow X$ with $f(0)=\zeta$ and $f(n) R f(n+1)$ for all $n \in \omega$.

Theorem 3. Stone's Theorem cannot be proved from ZF + DC.

Proof. Observe that if $p_{0} \geqslant p_{1} \geqslant \cdots \geqslant p_{n} \geqslant \ldots$ for a sequence of $p_{n} \in \mathbf{P}$, then there is a $q \in \mathbf{P}$ with $q \leqslant p_{n}$ for all $n \in \omega$, namely $q=\bigcup_{n \in \omega} p_{n}$. We can now repeat the proof of Lemma 8.5 in $[6]$ to show that if $g \in \mathcal{M}[G]$ is a function on $\omega$ with values in $\mathcal{N}$, then $g \in \mathcal{N}$.

To complete the proof, suppose $X \in \mathcal{N}$ and $R \in \mathcal{N}$ is a relation on $X$, as in the hypothesis of DC. Then $X$ and $R$ are in $\mathcal{M}[G]$ and, using DC in $\mathcal{M}[G]$, given $\zeta \in X$ there is an $f \in \mathcal{M}[G], f: \omega \rightarrow X$, with $f(0)=\zeta$ and $f(n) R f(n+1)$ for all $n \in \omega$. It follows that $f \in \mathcal{N}$. Therefore Dependent Choice holds in $\mathcal{N}$.

Recall from [6] that $\mathrm{AC}$ is equivalent to $\forall \kappa \mathrm{DC}_{\kappa}$. By reproducing the proof of Theorem 1 using the partial order $\mathbf{P}=F n(\lambda \times \mathbf{R} \times \lambda \times \lambda, 2, \lambda)$ for regular $\lambda$ and by generating the normal filter with sets having support less than $\lambda$, we obtain a ZF model of $\forall \kappa<\lambda \mathrm{DC}_{\kappa}$ in which Stone's Theorem fails.

Notice that an essential part of the proof of Theorem 2 is the fact that every proper open subset of a connected space has a non-empty border.

Theorem 4. (ConZF) There exists a zero-dimensional metric space that is not paracompact.

Proof. Let $\mathcal{G}$ be the group of automorphisms of $B$ induced by permutations $\pi$ of $\omega \times \mathbf{R} \times \omega_{1}$ satisfying $\pi(n, r, \alpha)=\left(n, \sigma(r), \alpha^{\prime}\right)$, where $\pi(n, r,$.$) is a permutation on$ $\omega_{1}$ for fixed $n, r$ and $\sigma: \mathbf{R} \rightarrow \mathbf{R}$ is a translation by some rational value. Let $\mathcal{F}$ be the (normal) filter on $\mathcal{G}$ generated by $\left\{\right.$ fix $\left.(e): e \in\left[\omega \times \mathbf{R} \times \omega_{1}\right]^{<\omega}\right\}$, and $\mathcal{N}$ the natural symmetric model. 
In addition to the previous elements of $\mathcal{M}[G]$, consider the following sets:

$$
\begin{aligned}
Q_{n} & =\left\{X_{n r}: r \in \mathbf{Q}\right\}, \\
I_{n} & =\left\{X_{n r}: r \notin \mathbf{Q}\right\} .
\end{aligned}
$$

As $\sigma$ is a rational shift, the $Q_{n}$ and $I_{n}$ have symmetric names and hence are elements of $\mathcal{N}$.

Claim 4.1. There is no function $f \in \mathcal{N}$ such that $\operatorname{dom}(f)=\omega$ and for all $n \in \omega$, $\varnothing \neq f(n) \subsetneq Q_{n}$.

Proof. Follow the proof of Claim 1.3, noting that $r$ and $s$ can be chosen to be rational and $\pi \in \mathcal{G}$ can be constructed appropriately.

Notice that $Q=\bigcup\left\{Q_{n}: n \in \omega\right\} \in \mathcal{N}$.

Claim 4.2. $Q$ is a zero-dimensional metric space.

Proof. The metric on $\bigcup M$ induces a metrizable topology on $Q$. A symmetric name exists for the linear order on $R_{n}$, so each $R_{n}$ is linearly ordered in $\mathcal{N}$. The base of clopen sets $\left\{(x, y) \cap Q_{n}: x, y \in I_{n}, n \in \omega\right\}$ shows that $Q$ is zero-dimensional.

Claim 4.3. $Q$ is not paracompact.

Proof. Let $\mathcal{U}$ be the open cover consisting of all open intervals with endpoints in some $Q_{n}$. Suppose $\mathcal{V}$ were a locally finite open refinement of $\mathcal{U}$. For $x \in Q$, define the open set $V_{x}=\bigcap\{V \in \mathcal{V}: x \in V\}$, ord $(x)=|\{V \in \mathcal{V}: x \in V\}|$, and $\mathcal{V}^{\prime}=\left\{V_{x}: x \in X\right\}$. Let $S$ be the set of all $x \in Q$ satisfying

either (1) $x \in Q_{n}, \exists y \in Q_{n} \operatorname{ord}(x) \neq \operatorname{ord}(y)$ and $\forall z \in Q_{n} \operatorname{ord}(x) \leqslant \operatorname{ord}(z)$

$$
\text { or (2) whenever } x \in V \in \mathcal{V}^{\prime} \exists y \in V \forall u, v \in V d(x, y) \geqslant \frac{2}{3} d(u, v) \text {. }
$$

Let $S_{n}=S \cap Q_{n}$. If there are $x, y \in Q_{n}$ with $\operatorname{ord}(x) \neq \operatorname{ord}(y)$, then $S_{n}$ is a proper subset of $S_{n}$. If not, the sets in $\mathcal{V}^{\prime}$ meeting $Q_{n}$ will be pairwise disjoint, and hence $S_{n}$ will be a proper subset of $Q_{n}$, because $Q_{n}$ is densely ordered. In any case, we have a contradiction to Claim 4.1.

\section{FURTHER RESEARCH}

As we observed after Theorem 3, ZF $+\left(\forall \kappa<\lambda \mathrm{DC}_{\kappa}\right)$ does not imply Stone's Theorem, for any $\lambda$. It is natural to ask, therefore, whether other weakenings of AC imply Stone's Theorem:

Question. Does ZF+ BPI, OP or SP imply Stone's Theorem?

Recall from [9] that the Boolean Prime Ideal Theorem, the statement that every Boolean algebra has a prime ideal, is equivalent to Tychonoff's Theorem for compact Hausdorff spaces, which is equivalent to the existence of the Stone-Čech compactification for Tychonoff spaces and also to the Compactness Theorem of first-order logic. BPI implies the Ordering Principle, OP, the statement that every set can be linearly ordered. Note that the ZFC proof of Stone's Theorem in [12] almost follows through using only $\mathrm{ZF}+\mathrm{OP}$. The only part of the argument which does not extend to this system is in showing that the locally finite refining collection of open sets covers the space.

The Selection Principle states that for every family of sets $\mathcal{F}$ with at least two elements there is a function $f$ such that for each $F \in \mathcal{F}, \varnothing \neq f(F) \subsetneq F$. SP follows 
from AC and implies OP [6]. Clearly SP fails in any model where Stone's Theorem is made to fail in the way we have devised here.

As indicated by the referee, the Axiom of Choice for infinite sets of pairs, $C(\infty, 2)$, fails in our models, and hence so do each of BPI, OP and SP, since these all imply $C(\infty, 2)$. For instance, to see that $C(\infty, 2)$ fails in the model of Theorem 4 we argue as follows:

If $A$ is any subset of $Q_{n}$, then $\operatorname{fix}((n, 0, \alpha)) \subseteq \operatorname{sym}(\underline{A})$ for any $\alpha$, since $\pi(n, r, \alpha)=$ $\left(n, r, \alpha^{\prime}\right)$ for any $\pi \in \operatorname{fix}((n, 0, \alpha))$. Hence every subset of every $Q_{n}$ is in $\mathcal{N}$. Now if $\mathcal{P}$ is the set of unordered pairs $\left\{\{C, D\}: C, D \subseteq Q_{n}\right.$ for some $\left.n, C \neq D\right\}$, then $\operatorname{sym}(\underline{\mathcal{P}})=\mathcal{G}$, so $\mathcal{P} \in \mathcal{N}$. Suppose that $f$ were a choice function for $\mathcal{P}$ with symmetric name $f$, and let $e$ be a finite subset of $\omega \times \mathbf{R} \times \omega_{1}$ such that $f i x(e) \subseteq \operatorname{sym}(f)$. Pick $n \in \bar{\omega}$ such that $e \cap\left(\{n\} \times \mathbf{R} \times \omega_{1}\right)=\varnothing$, and let $O_{n}=\left\{X_{n, 2 i+1}: i \in \mathbf{Z}\right\}$ and $E_{n}=\left\{X_{n, 2 i}: i \in \mathbf{Z}\right\}$. Then, without loss of generality, there exists some $p$ such that

$$
p \Vdash(\underline{f} \text { is a function }) \text { and }\left(\underline{f}\left(\left(\underline{O}_{n}, \underline{E}_{n}\right)\right)=\underline{O}_{n}\right) .
$$

With $\epsilon$ and $\phi$ as in Claim 1.3, define $\pi \in \mathcal{G}$ by

$$
\pi(m, t, \alpha)= \begin{cases}(m, t, \alpha), & m \neq n, \\ (n, r+1, \phi(\alpha)), & m=n, \alpha \in[0, \epsilon], \\ \left(n, r+1, \phi^{-1}(\alpha)\right), & m=n, \alpha \in(\epsilon, 2 \epsilon], \\ (n, r+1, \alpha), & m=n, \alpha>2 \epsilon .\end{cases}
$$

Now, as in 1.3, $\pi(\underline{f})=\underline{f}, \pi\left(\underline{O}_{n}, \underline{E}_{n}\right)=\left(\underline{O}_{n}, \underline{E}_{n}\right)$, and $\pi p$ and $p$ are compatible. So

$$
\pi p \cup p \Vdash\left(\underline{f}\left(\left(\underline{O}_{n}, \underline{E}_{n}\right)\right)=\underline{O}_{n}\right) \text { and }\left(\underline{f}\left(\left(\underline{O}_{n}, \underline{E}_{n}\right)\right)=\underline{E}_{n}\right),
$$

a contradiction, completing the argument.

On the other hand, Proposition 5 below shows that all proofs of Stone's Theorem (known to the authors) actually prove a stronger conclusion which implies AC. It is based on an idea from [1]. Let us call a refinement $\mathcal{V}$ of $\mathcal{U}$ effective if there is a function $a: \mathcal{V} \rightarrow \mathcal{U}$ such that $V \subseteq a(V)$ for all $V \in \mathcal{V}$. (We do not require that each $a(V)$ be non-empty, but only that $\mathcal{V}$ covers). Let us also say that a space is effectively metacompact if every open cover has an effective point-finite open refinement.

Proposition 5. (ZF) If every discrete metric space is effectively metacompact, then the Axiom of Choice holds.

Proof. Let $\mathcal{F}$ be any family of disjoint non-empty sets and let $X=\mathcal{F} \cup \cup \mathcal{F}$ have the discrete metric. Let $\mathcal{V}$ be an effective point-finite open refinement of the open cover $\mathcal{U}=\{\{x, F\}: x \in F \in \mathcal{F}\}$, with associated function $a$. For each $F \in \mathcal{F}$, let $C(F)=\{V: F \in V\}$. Now each $C(F)$ is finite and non-empty, so $f(F)=\{x:\{x, F\}=a(V), V \in C(F)\}$ is a finite and non-empty subset of $F$, for each $F \in \mathcal{F}$.

Thus the Axiom of Multiple Choice holds for an arbitrary collection of non-empty pairwise disjoint sets, $\mathcal{F}$, but $\mathrm{MC}$ implies $\mathrm{AC}$ in $\mathrm{ZF}[6,9.1]$.

Finally, it is possible to work with models of set theory with atoms, ZFA, to construct non-paracompact metric spaces. With reference to the question above, Mostowski has shown in [8] that SP is false in every model in which the set of atoms cannot be well ordered. 


\section{ACKNOWLEDGEMENT}

The authors would like to thank the referee for demonstrating that $C(\infty, 2)$ fails in the models here, and for other improvements to this paper.

\section{REFERENCES}

1. Brunner N., The axiom of choice in topology, Notre Dame J. Formal Logic 24 (1983), 305-317. MR 85j:54057

2. Dieudonné J.A., Une généralisation des espaces compacts, J. Math. Pures. Appl. 23 (1944), 65-76. MR 7:134f

3. van Douwen E.K., Horrors of topology without AC: a nonnormal orderable space, Proc. Amer. Math. Soc. 95 (1985), 101-105. MR 87d:03130

4. Good C. and Tree I. J., Continuing horrors of topology without choice, Top. Appl. 63 (1995), 79-90. MR 96f:54003

5. Howard P.E., Rado's selection lemma does not imply the Boolean Prime Ideal Theorem, Z. Math Logic Grundlag Math. 30 (1984), 129-132. MR 85d:03099

6. Jech T., The Axiom of Choice, North Holland, Amsterdam 1973. MR 53:139

7. Kunen K., Set Theory, An Introduction To Independence Proofs, North Holland, Amsterdam 1980. MR 82f:03001

8. Mostowski A., On a problem of W. Kinna and K. Wagner, Colloq. Math. 6 (1958), 207-208. MR 20:6980

9. Potter M. D., Sets, An Introduction, Oxford University Press, New York 1990. MR 92c:04001

10. Rudin M. E., A new proof that metric spaces are paracompact, Proc. Amer. Math. Soc. 20 (1969), 603. MR 38:5170

11. Stone A.H., Paracompactness and product spaces, Bull. Amer. Math. Soc. 54 (1948), 977-982. MR 10:204c

12. Willard S., General Topology, Addison-Wesley 1970. MR 41:9173

School of Mathematics and Statistics, University of Birmingham, Edgbaston B15 2TT, ENGLAND

E-mail address: c.good@bham.ac.uk

62 Arle Gardens, Cheltenham, Gloucestershire Gl51 8HR, England

Department of Mathematics, York University, North York, Ontario, Canada M3J $1 \mathrm{P} 3$

E-mail address: watson@mathstat.yorku.ca 